\title{
Super-resolution microscopy and its applications in neuroscience
}

\author{
Xuecen Wang, ${ }^{*,}$, Jiahao Wang*, ${ }^{*}$ Xinpei Zhu*,§, Yao Zheng ${ }^{\dagger}, \mathrm{Ke} \mathrm{Si}^{*, \dagger}$ and Wei Gong** \\ *Institute of Neuroscience, Department of Neurobiology \\ Key Laboratory of Medical Neurobiology of the Ministry of \\ Health of China, Zhejiang Province Key Laboratory \\ of Neurobiology, Zhejiang University, School of Medicine \\ Hangzhou, Zhejiang 310058, P. R. China \\ ${ }^{\dagger}$ State Key Laboratory of Modern Optical Instrumentation \\ College of Optical Science and Engineering, and \\ the Collaborative Innovation Center for Brain Science \\ Zhejiang University, Hangzhou, Zhejiang 310027, P. R. China \\ *weigong@zju.edu.cn
}

Received 16 December 2016

Accepted 23 February 2017

Published 22 March 2017

\begin{abstract}
Optical microscopy promises researchers to see most tiny substances directly. However, the resolution of conventional microscopy is restricted by the diffraction limit. This makes it a challenge to observe subcellular processes happened in nanoscale. The development of superresolution microscopy provides a solution to this challenge. Here, we briefly review several commonly used super-resolution techniques, explicating their basic principles and applications in biological science, especially in neuroscience. In addition, characteristics and limitations of each technique are compared to provide a guidance for biologists to choose the most suitable tool.
\end{abstract}

Keywords: Super-resolution microscopy; total internal reflection fluorescence microscopy; stimulated emission depletion microscopy; structure illumination microscopy; photoactivation localization microscopy; stochastic optical reconstruction microscopy.

\section{Introduction}

Optical microscopy is one of the key, versatile and powerful tool in scientific researches, especially in biology. Together with a series of techniques, optical microscopy facilitated the appearance and development of modern biology such as cell biology and microbiology. Millstone was the development of fluorescence microscopy, which made it much easier

\$Corresponding author.

$\S_{\text {Those authors contributed equally to the work. }}$

This is an Open Access article published by World Scientific Publishing Company. It is distributed under the terms of the Creative Commons Attribution 4.0 (CC-BY) License. Further distribution of this work is permitted, provided the original work is properly cited. 
to obtain multi-color information of the distribution and morphology of cells.

Conventional fluorescence microscope collects fluorescence emitted by fluorophores through an objective lens. In fact, when light passes through an objective lens, it will form a hazy spot in the image plane, instead of an infinitesimal point, due to diffraction. The resolution of a microscope is dependent on the intensity profile of this spot, which is called point spread function (PSF). The full width half maximum (FWHM) of the PSF (called as the 'diffraction limit') representing the highest resolution is determined by the numerical aperture of the objective lens and the wavelength of the light. Abbe found the formula of the diffraction limit for the first time in 1873 and calculated its value, which was approximately $200-300 \mathrm{~nm}$ in the lateral direction $(x, y)$ and $500-700 \mathrm{~nm}$ in the axial direction $(z)$ for visible incident light. This resolution is sufficient to image in tissue or cellular level. However, it is still not high enough to solve vast majorities of questions in subcellular level. Thus, scientists have devoted themselves to developing imaging techniques which aim to break the diffraction limit. These include near-field optical microscopes which shorten the prolongation distance of emitted light, thus obtaining a better axial resolution, since light only propagates a distance less than its wavelength without spatial divergence. ${ }^{1,2}$ Besides, standing wave fluorescence microscopy (SWFM), ${ }^{3}$ 4-Pi confocal microscopy ${ }^{4}$ and I5M microscopy ${ }^{5}$ apply the interference of light to improve the axial resolution up to $100 \mathrm{~nm}$. Nevertheless, the near-field imaging is confined to surfaces because of its short working distance. For the conventional far-field microscopies, such as confocal microscopy and two-photon microscopy, the working distance can be extended to millimeter. However, their resolution is restricted by diffraction limit, which is not enough for subcellular researches.

More recently, some novel far-field super-resolution fluorescence microscopies have been developed to break through the diffraction limit. These microscopies include stimulated emission depletion microscopy (STED), ${ }^{6}$ (saturated) structured illumination microscopy ((S)SIM $)^{7,8}$ based on patterned illumination, and (fluorescence) photoactivation localization microscopy ((F)PALM $)^{9,10}$ or stochastic optical reconstruction microscopy $(\mathrm{STORM})^{11}$ relying on single molecule localization.
The development of super-resolution microscopy offers a promising tool to resolve many fundamental problems in biological science. In the following sections, we will discuss the above commonly used super-resolution microscopies, including TIRFM, STED, SIM and (F)PALM/STORM. We will briefly present the principles, summarize their features and differences. Besides, their applications in biological science, especially in neuroscience, will also be discussed.

\section{Total Internal Reflection Fluorescence Microscopy (TIRFM)}

TIRFM is the typical near-field super-resolution microscopy by employing the evanescent field to excite the fluorescence molecules close to the sample surface, and then the features or events of interests are captured. ${ }^{12}$ Therefore, TIRFM is also called evanescent field microscopy.

Classical optics explicates that when light spreads from medium A to medium B, it will both reflect and refract on the interface at the same time. However, there is a special case called total internal reflection, when light transmits from an optically denser medium A to thinner medium B, it will totally reflect back to medium when its incidence angle is bigger than the critical angle, as illustrated in Fig. 1(a). Under this situation, although no light refracts into medium $\mathrm{B}$, a small portion of the light energy will still propagate into medium B and form an 'evanescent field.' The intensity of the evanescent field decays exponentially with the increasing of spread distance (Fig. 1(b)). Typically, TIRFM is merely able to stimulate the fluorescent probes and proteins within the range of $200 \mathrm{~nm}$ close to the interface, which avoids the excitation of fluorophores farther in the cells. As a result, TIRFM can dramatically increase the signal-to-noise ratio and the spatial resolution in the axial direction. In practice, researchers use TIRFM to observe cells growing on glass slides. In this case, light transmitting from glass to water will totally internally reflect in the glass-water interface, forming the evanescent field in the thin layer close to the glass slides and ultimately achieving high resolution images.

TIRFM becomes a powerful and popular approach, for addressing the tiny structures and dynamic processes at or near the plasma membrane of cells that are immediately adjacent to the cover 


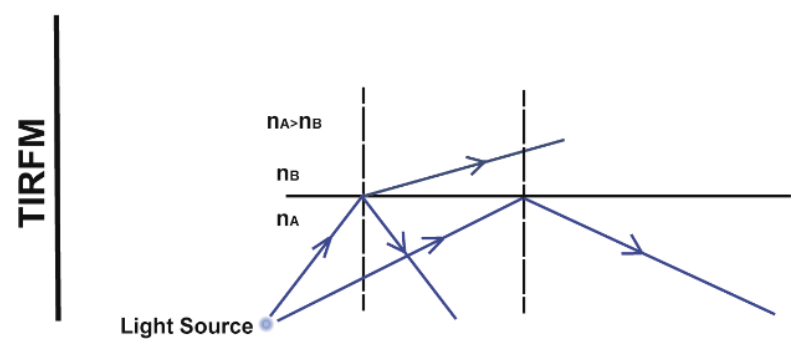

(a)

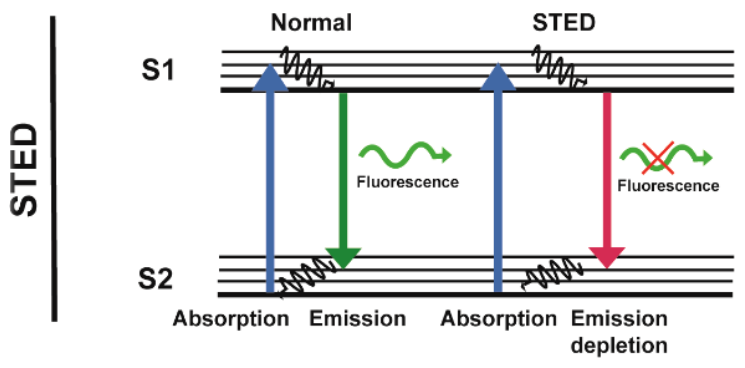

(c)


(e)
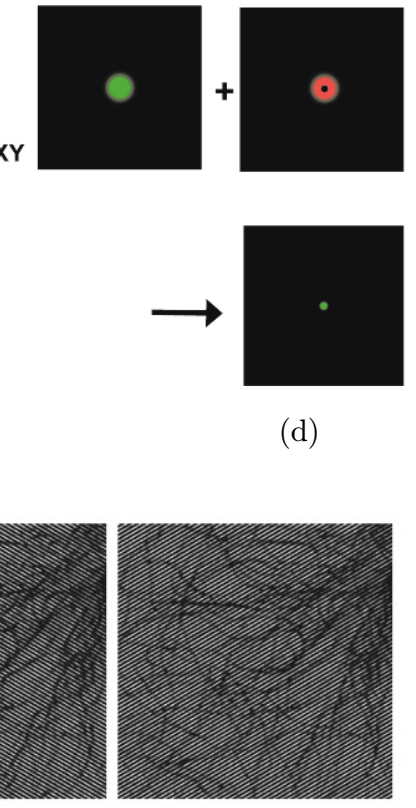

(d)

(b)
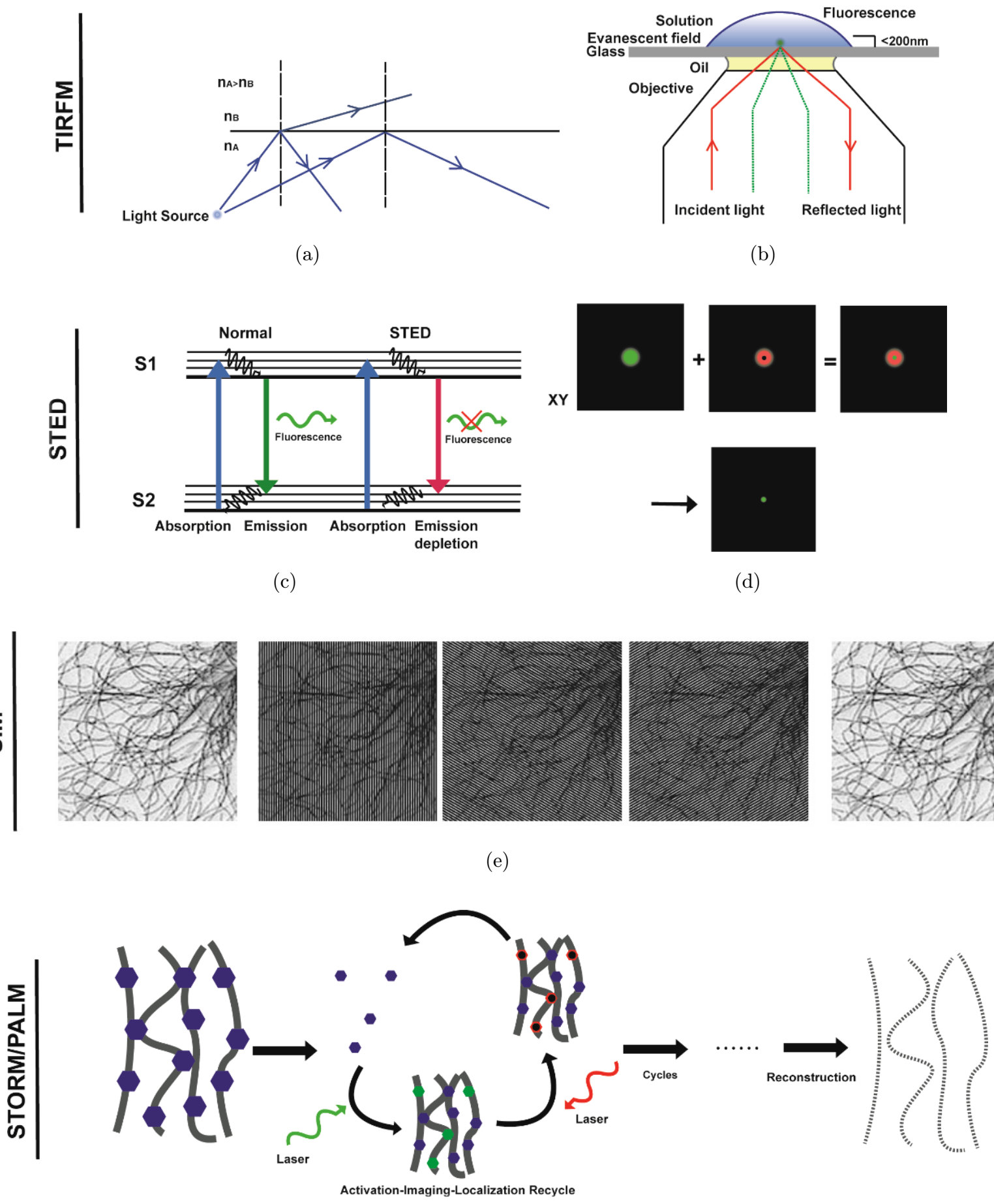

(f)

Fig. 1. Schematic diagram of principles of TIRFM, STED, SIM and (F)PALM/STORM. (a) As light spread from medium A to medium $\mathrm{B}\left(n_{\mathrm{A}}>n_{\mathrm{B}}\right)$, it reflects and refracts at the same time. But it will totally reflect when its incident angle is larger than the critical angle; (b) Under the case of total internal reflection, fluorescence is emitted by evanescent field and collected by the objective lens; (c) Normally, fluorophores jump from the ground state (S2) to the excitation state (S1) after illumination, then return to the ground state and release fluorescence at the same time. However, the depletion beam interrupts this process and depletes the spontaneous fluorescence; (d) The doughnut-like depletion beam quench the spontaneous fluorescence around the central point, forming a smaller effective PSF; €Images are reconstructed by extracting the high frequency information, shifting it to low frequency, and then taking images at different grating angles and phases; (f) Schematic diagram of (F)PALM/STORM. A small number of discrete fluorophores are detected stochastically at one moment. After a lot of cycles, the whole image is recovered with precise algorithms based on the location of each fluorophore. 
glass. Indeed, the decipherment of vesicle transport certainly benefit from the use of TIRFM as the vesicles and involved molecules could be detected actively and spatially precisely. ${ }^{13}$ It is worth mentioning that TIRFM employs lower intensity of power, resulting in less damage to live cell samples. So TIRFM is suitable to monitor the dynamic processes of signaling pathway, endocytosis, and exocytosis for a long time. ${ }^{14-16}$ However, it was difficult to distinguish multi-color information, which limited its applications in researches of dynamic interactions between proteins. Recently, Yuan et al. successfully visualized elementary exocytosis-coupled clathrin-mediated endocytosis in insulin-secreting INS-1 cells by TIRFM combing dual-color with image processing techniques. ${ }^{17}$ Moreover, a method using multi-angle TIRFM to reconstruct $3 \mathrm{D}$ image sequences was reported with an axial resolution up to $50-100 \mathrm{~nm}$ on average in cellular context. ${ }^{18}$

Besides, TIRFM could combine with other fluorescence microscopes easily, such as EPI fluorescent microscope, because both of them are wide-field microscopes. The trafficking of glutamate transporters by VAMP3 vesicles in astrocytes was described with EPI-TIRF directly. ${ }^{19}$ The illumination of EPI could come from either an independent light source ${ }^{20}$ or the incidence light of TIRFM. Besides EPI-TIRF, other imaging system, such as the fast scanning confocal microscopy using a resonance scanner or spiking disk, can also be combined with TIRFM, resulting in an improvement in axial resolution. ${ }^{21}$

Nevertheless, there are still some limitations existing in the TIRFM application. Note that the evanescent field is restricted to $\sim 200 \mathrm{~nm}$ above the glass slide, meaning that specimens are required to tightly adhere to the glass. Besides, how to maintain the activity of live samples and the stability of focus spot during long-time imaging in practice needs to be considered.

\section{Stimulated Emission Depletion (STED) Microscopy}

STED is a far-field microscopy, based on the confocal microscopy, by using the stimulated excitation phenomenon. Its principle is illustrated in Figs. 1(c) and 1(d). For the exclusive contributions on superresolution microscopy, Hell got the Nobel Prize in 2014. He proposed the concept of STED microscopy for the first time in 1994, ${ }^{6}$ and experimentally proved its operability in $1999 .{ }^{22}$ Usually, when a fluorophore is excited by the excitation beam, it jumps from the ground state to the excited state. After that, it turns back to the ground state, accompanying with spontaneous fluorescence. Conventional fluorescence microscopes collect all these spontaneous fluorescence to form a diffraction-limited image, yet a different strategy has been adopted in STED microscopy. The key technique is that STED microscopy uses a second laser beam, called depletion beam, to interrupt the transition processes and manipulate the sample's fluorescence emission. The depletion beam is powerful enough to bring a fluorophore from the excited state to the ground state before it emits spontaneous fluorescence, leaving a center focal spot active to emit spontaneous fluorescence. Thus, it minimizes the area of illumination at the focal point. In another word, STED microscopy employs two coaxial lasers at the same time: one is used as the excitation beam to excite the spontaneous fluorescence of samples; the other one is a doughnut-pattern depletion beam that suppresses spontaneous fluorescence emission surrounding the center focal spot of the excitation beam. Therefore, the region of the spontaneous fluorescence is smaller than the airy disk, which represents smaller PSF, leading to an enhanced resolution. A point-by-point or parallelized scanning scheme is used to generate a super-resolved image with STED microscopy. A more general STED-like approach RESOLFT, named after 'reversible saturable optical fluorescence transition', utilizes the transitions of reversible photoswitchable fluorescent proteins or fluorophores between an activated and a nonactivated state. Hence, lower intensity of light is used to image, resulting in less photodamages. $^{23}$

STED microscopy wipes out the fluorescence interference from off-target fluorophores with a donut-shaped depletion beam whose intensity is above the fluorophore saturation level. It is noteworthy that unlimited resolution improvement is allowed with this approach in principle. But in practice, the resolution is restricted by many factors, such as the power of the depletion laser, photobleaching, and aberrations in the optics. Two main aspects which may limit the applications of STED microscopy have drawn the attention of scientists: one is to produce the doughnut-shaped depletion beam and align it coaxially with the 
excitation beam using a complex control system; the other is its limited penetration depth. Marsh et al. simplified the system by integrating the excitation beam and the depletion beam to an inverted confocal fluorescence lifetime imaging microscopes (FLIM) and using the time difference to achieve super-resolution up to $100 \mathrm{~nm} .{ }^{24}$ Besides, Kuang et al. proposed a STED-like system, called fluorescence emission difference microscopy (FED), by subtracting two images scanning with two different point spread functions. ${ }^{25}$ Notably, FED is different from STED because it bases on the difference of intensity between two different images. In order to increase imaging depth, deep tissue STED microscopy was reported by using a Gaussian beam for excitation and a hollow Bessel beam for depletion. ${ }^{26}$ Consistent super-resolution imaging was achieved up to $155 \mu \mathrm{m}$ deep in a solid agarose sample and $100 \mu \mathrm{m}$ deep in a phantom of gray matter in brain tissue. Recently, Xi et al. narrowed the axially PSF of STED by employing the interference between the $\mathrm{PSF}$ and its reflection from a mirror placed behind the specimen. ${ }^{27}$

Despite its short time from the invention, STED microscopy has made many contributions to the observation of subcellular structures such as endoplasmic reticulum, ${ }^{28}$ mitochondria ${ }^{29,30}$ and cytoskeletons. ${ }^{31,32}$ Because of complicated components involved in signal transduction between cells, dualcolor $\mathrm{STED}^{33-35}$ and even multi-color $\mathrm{STED}^{36,37}$ have been established to study several active complexes at the same time. Besides, 3D STED is also available for biologists which provides $3 \mathrm{D}$ imaging with the resolution of $45 \times 45 \times 108 \mathrm{~nm}$ in a cell originally. ${ }^{38}$

Admittedly, although STED has wide application, the intensity of depletion beam is too high that photobleaching of fluorophores and photodamage to specimens could be induced within short time. ${ }^{39}$ This property mainly limits the long-time use of STED in live samples.

\section{Structure Illumination Microscopy (SIM)}

Another commonly used far-field super-resolution microscopy is called structure illumination microscopy (SIM). It breaks the diffraction limit by illuminating a sample with patterned light based on a standard wide-field fluorescence microscope.
The excitation beam exhibits a periodically spatial pattern, ${ }^{8}$ which is originally a sinusoidal grid that can be generated by a digital micromirror device, ${ }^{40}$ replacing the conventional uniform illumination. The spatial frequency of the excitation light mixes with the specimen, shifting high frequency information of the sample into lower frequency which then could be detected by the microscope. Finally, super-resolution images can be reconstructed by capturing the low frequency information and recording images with different orientations and phases. The schematic figure for SIM is demonstrated in Fig. 1(e). Since SIM is based on a standard wide-field microscope, it could achieve a broader field of view in less time, compared with the scanning microscopy such as STED. However, the structure illumination pattern itself is restricted by the diffraction limit, resulting in only 2 -fold improvements in resolution, approximately $100 \mathrm{~nm}$ in the lateral direction and $300 \mathrm{~nm}$ in the axial direction.

In 2005, Gustafsson modified SIM with nonlinear structure illumination, which is named as saturated structure illumination microscopy (SSIM). ${ }^{8}$ The resolution with SSIM in the lateral direction can be achieved up to approximately $50 \mathrm{~nm}$. Instead of the traditional sinusoidal grid, SSIM employs a very high intensity of excitation light to saturate the fluorophores. Therefore, in the linear region, the emitted fluorescence intensity is proportional to the power of the incident light; while in the saturation region, the fluorescence emission remains in a stable saturation level even when the intensity of illumination increases persistently. If the strong illumination light has a sinusoidal pattern, fluorescence saturation would clip the peaks of the excitation pattern, making it flat. Thus, the resolution of SSIM is limited by the level of fluorescence saturation, instead of diffraction limit.

Nowadays, SIM is widely used to image the living samples as it employs illumination light with low intensity. Reqo and Gustafsson successfully reduced the excitation intensity six times lower than that needed for saturation, ultimately imaging the purified microtubule with a resolution up to $50 \mathrm{~nm} .{ }^{41}$ The extended resolution has also been achieved with the help of high NA lens, reaching approximately 45$62 \mathrm{~nm} .^{42}$ Besides, good compatibility with many kinds of fluorescence probes made it possible to catch dual-color information or multi-color images of three dimensions. Currently, a high-throughput dual-color 
precision imaging technique, based on SIM, was reported to achieve the imaging data of multiple types of neurons and projections in a whole mouse brain at single cell resolution. ${ }^{43}$ One point which may contribute to better images is to increase the photostability and brightness of the fluorophore since the clear detection and modulation of fluorescence is based on a stable and vigorous fluorescence signal.

\section{Photoactivation Localization Microscopy (PALM)/Stochastic Optical Reconstruction Microscopy (STORM)/Fluorescence Photoactivation Localization Microscopy (FPALM)}

Most super-resolution microscopy achieves super resolution by decreasing the size of the effective PSF. However, Betzig, Hess, and Zhuang reconstructed super-resolution images depending on the localization of single molecule and named them as PALM,${ }^{9}$ FPALM,${ }^{10}$ and STORM,${ }^{11}$ respectively. Samples which are marked with fluorophores usually contain millions of fluorescence molecules. Each molecule presents as a point light source and exhibits as an airy disk pattern after passing through the imaging system. However, those fluorophores are usually densely packaged, thus their images overlap and are unable to be distinguished from each other. (F)PALM and STORM were developed to distinguish the fluorophores by exciting them at a different time and then calculate their spatial localization accurately using a Gaussian filter. Figure 1(f) displays a general diagram of the image caption.

Fluorescent proteins or dyes used in (F)PALM/ STORM are regulated by an excitation light. The fluorescent proteins or dyes can switch between the excitation state and the dark state. At any moment, the majority of fluorophores in samples are in the dark state and only a few of them are in the excitation state stochastically, which are sparsely distributed in the sample and thus can be localized accurately. Then the excited fluorophores are deactivated permanently and another subset of fluorophores are excited and localized. After numerous repeats of this process, the super-resolution image of the specimen is reconstructed with the exact localization of each fluorophore. The key point of (F)PALM/STORM is the employment of photoswitched fluorescence probes which are able to switch between the excitation state and the dark state. Generally, there are two groups of fluorescent probes: fluorescent proteins (FPs) and nongenetically encoded probes. Most FPs used in $(\mathrm{F})$ PALM/STORM are derived from common FPs, such as green fluorescent protein (GFP). Genetic tools are used to change specific properties of common FPs to give them the ability to switch. PAmcherry, EYFP, and PA-GFP, frequently used in experiments, always have a better compatibility with biological samples in vivo. Compared with FPs, nongenetically encoded probes, such as Alexa 647 and Cy7, usually have much more fluorescent intensity than FPs but are more toxic to biological specimens. Several strategies have been described to develop better fluorescent probes for live-cell imaging. ${ }^{44,45}$ As each of the fluorescent probes has advantages and disadvantages, scientists should apply suitable fluorescent probe according to the experiments and samples.

So far, (F)PALM/STORM has been used in the study of microtubules, ${ }^{46}$ microfilaments,${ }^{9}$ adhesion complexes and other subcellular structures. 3D STORM also plays an important role in analyzing the organization of chromatin in transcriptional active state. ${ }^{47}$ When it comes to living cells, it is shown that the spatial resolution could achieve 20-40 $\mathrm{nm}$ while keeping the temporal resolution at $0.5-10 \mathrm{~s}^{48}$ As (F)PALM/STORM greatly depends on fluorophores, by combing biomolecular fluorescence complementation and PALM, Liu et al. successfully imaged the location and dynamics of MreB and Ef-Tu in Escherichia coli cell. ${ }^{49} \mathrm{Li}$ et al. reported a trident perylenemonoimide dye modified by triple dithienylethenes which has high sensitivity of on/off switching and with its help, images of vesicles within sub-100 nm resolution can be achieved. ${ }^{44}$ Pan et al. developed organic fluorescent probes, which are not only cell permeable but also targeting endogenous proteins specifically. They successfully dissected the dynamics of $\mathrm{F}$-actin whose diameter is approximately $80 \mathrm{~nm}$ with $10 \mathrm{~s}$ temporal resolution in live cells. ${ }^{45}$

For (F)PALM/STORM, fluorescent molecules and staining methods should be chosen with caution, as the techniques are inefficient when there are too many molecules in samples. ${ }^{50,51}$ In addition, limited imaging speed hinders its applications in living cell researches, though a time resolution of $3 \mathrm{~s}$ has been achieved.$^{52}$ Besides, complicated procedures 
Super-resolution microscopy and its applications in neuroscience

and post-imaging processing may be a problem for biologists who are not familiar with the optical setup and algorithm.

\section{Applications in Neuroscience}

For neurosciences, the dynamic process of vesicles forming, transferring and involved proteins have been discussed for a long time because it is always recognized as the fundamental unit of neural network communication. What's more, neuronal basic structure and morphology, which plays an essential role in synaptic plasticity and disease of nervous system, are also concerned by scientists. Currently, most of the above understanding came from conventional optical microscopy, such as the localization of key molecular and the rough morphological changes, but the precise process and small protein complex component (length scales are usually below $200 \mathrm{~nm}$ ) are still unclear. Thanks to the invention of super-resolution microscopy, it opens up the giant dream for monitoring these mysteries.

Neural signals are passed from one neuron to another by synapse. This process named synaptic transmission has drawn attention of the scientists for ages. ${ }^{53}$ Early studies only recognized correlated proteins' distributions, but have no idea of its dynamics. More recently, with the help of STED microscopy, vesicle movements and protein trafficking have been investigated. Willig et al. investigated the vesicles after fusion and found that synaptotagmin-1, one of the vesicle membrane proteins residents, remained clustered in cell membrane following vesicle endocytosis. They suggested that during vesicle recycling, some vesicle components still remained clustered. ${ }^{54}$ Subsequently, other prominent presynaptic protein clustering such as syntaxin were also found. ${ }^{55}$ With the development of high imaging speed STED in 2008, the single vesical moving speed was detected, which revealed that vesicles move more rapidly outside of boutons than within boutons. ${ }^{56}$ Meanwhile, Nofal et al. devised a new method to analyze the mobility of vesicles and distinguished them when vesicles were docked to the cell membrane using TIRFM. ${ }^{57}$

Another line is investigating post-synaptic phenomena, in particular, the receptors and spines. Using TIRFM, Gu et al. directly visualized individual exocytic events of NMDA receptors in rat hippocampal neurons and found that the constitutive exocytosis of NMDA receptors was mediated by
SNAP25-VAMP1-syntaxin4 complex. ${ }^{58}$ Nägerl et al. demonstrated that the dendritic spines can be tracking long time for the first time in living cell using STED. ${ }^{59}$ What's more, Schouten et al. also identified dendritic spines of rat primary hippocampal neurons using SIM and analyzed the spines morphology quantitatively. ${ }^{60}$

Because of the characteristics of PALM/STORM algorithm, this super-resolution microscopy is convenient for monitoring the structure of axons. Zhuang et al. used STORM to observe the development of axons and revealed the mechanism of actin, spectrin, and associated molecules forming a periodic sub-membrane lattice structure in axons. ${ }^{61}$ Meanwhile, they also noticed the distribution of sodium channels in axons is in a periodic pattern, which means that this distribution may also be related to the periodic cytoskeletal structure in axons. ${ }^{62}$

Despite super-resolution microscopies receiving great attention, these advanced techniques have not been chosen by many neuroscience groups up to now. But with the development, there will be more and more probabilities for super-resolution microscopies applied in the neuroscience field in future.

\section{Conclusion and Outlook}

Over the past two decades, super-resolution microscopy has developed quickly from an original concept to a series of sophisticated imaging techniques surpassing the diffraction limit of light in various aspects. These innovative technologies have enabled scientists to investigate biological structures and physiological functions with better resolution in live samples than ever before.

In order to choose appropriate tools in different biological experiments, it is important to recognize the advantages and disadvantages of each approach. As a typical near-field microscope, TIRFM makes an improvement in the resolution of axial direction instead of lateral direction but have a relatively shorter working distance. On the other hand, far-field microscopes such as STED, (S)SIM and (F)PALM/STORM, which have been reviewed above, improve the resolution in the lateral direction and sometimes in the axial direction. STED microscopy is able to achieve satisfactory images but samples are at the risk of being damaged by the high power lasers. Compared with STED microscopy, SIM and SSIM make fewer improvements in the lateral resolution. However, SIM and SSIM are 
X. Wang et al.

Table 1. Comparison of super-resolution microscopy.

\begin{tabular}{|c|c|c|c|c|c|}
\hline Approach & Confocal microscopy & TIRFM & STED & (S)SIM & (F)PALM/STORM \\
\hline$x-y$ resolution $(\mathrm{nm})$ & $200-250$ & $100-200$ & $20-70$ & $80-100 \sim 50($ SSIM $)$ & $10-40$ \\
\hline$z$ resolution $(\mathrm{nm})$ & $500-700$ & $20-50$ & $30-100$ & $250-300$ & $10-50$ \\
\hline Imaging speed & ms-s & ms-s & ms-s & ms-s & $\mathrm{s}-\mathrm{min}$ \\
\hline Phototoxicity & + & + & +++ & + & +++ \\
\hline Live cell imaging & +++ & +++ & + & +++ & + \\
\hline Multi-color imaging & ++ & + & ++ & +++ & +++ \\
\hline 3D imaging & +++ & + & ++ & ++ & ++ \\
\hline Sample requirement & Broad & $\begin{array}{l}\text { Tightly adhere } \\
\text { to glass slide }\end{array}$ & $\begin{array}{l}\text { Photostable } \\
\text { fluorophores }\end{array}$ & $\begin{array}{c}\text { Strong and stable } \\
\text { fluorophores }\end{array}$ & $\begin{array}{c}\text { Sparse photoswitchable } \\
\text { fluorophores }\end{array}$ \\
\hline
\end{tabular}

Note: Values are taken from the following Refs. 37, 42 and 67-70.

excellent tools for live cell imaging because of low illumination intensities required, great compatibility with various fluorescent probes and fast imaging speed with large a field of view. (F)PALM/STORM may perform better in making a balance between spatial and temporal resolution, as well as multicolor imaging. But an efficient fluorescent labeling method is urgently required. Besides, since (F) PALM/STORM locates the fluorophore statistically, it is most suitable to the fibrous structure such as axons. More detailed information of each microscopy is summarized in Table 1.

Further improvements of super-resolution microscopy may focus on the following three aspects: spatial resolution, temporal resolution, and imaging depth. First, the spatial resolution of these novel imaging methods, which in principle could approach to infinitely high, could be further improved by enhancing system signal-to-noise ratio, and fluorophores quantum efficiency. Alternatively, the dipole orientation information extracted from fluorescent polarization could also contribute to the enhancement of image resolution. ${ }^{63}$ Second, the imaging speed should be accelerated to catch instantaneous molecular biological activities. Third, the depth of super-resolution microscopy calls for an innovative improvement. Currently, super-resolution techniques are only suitable for observing the superficial surface of the sample. As the depth of sample increases, scattering and accumulated optical aberration cannot be neglected, which will dramatically degraded the imaging resolution. To overcome this problem, adaptive optics may be one of the potential solutions. ${ }^{64-66}$ In addition, the design of fluorescent probes with higher quantum efficiency, less photobleaching, and smaller sizes will notably improve the resolution. We expect that further development of super-resolution microscopy will yield efficient and critical insights into biological science.

\section{Acknowledgment}

We acknowledge support from National Basic Research Program of China (973 Program) (2015CB352005), National Natural Science Foundation of China (No. 6142780065, 31571110, 81527901), Natural Science Foundation of Zhejiang Province of China (No. Y16F050002) and the Fundamental Research Funds for the Central Universities.

\section{References}

1. E. A. Ash, G. Nicholls, "Super-resolution aperture scanning microscope," Nature 237, 510-512 (1972).

2. E. Betzig, J. K. Trautman, "Near-field optics: Microscopy, spectroscopy, and surface modification beyond the diffraction limit," Science 257:189-95 (1992).

3. B. Bailey, D. L. Farkas, D. L. Taylor et al., "Enhancement of axial resolution in fluorescence microscopy by standing-wave excitation," Nature 366, 44-48 (1993).

4. S. W. Hell, E. H. Stelzer, S. Lindek et al., "Confocal microscopy with an increased detection aperture: Type-B 4Pi confocal microscopy," Opt. Lett. 19, 222 (1994).

5. M. G. Gustafsson, D. A. Agard, J. W. Sedat, "I5M: 3D widefield light microscopy with better than 100 nm axial resolution," J. Microsc. 195, 10-16 (1999).

6. S. W. Hell, J. Wichmann, "Breaking the diffraction resolution limit by stimulated emission: Stimulatedemission-depletion fluorescence microscopy," Opt. Lett. 19, 780-782 (1994). 
7. R. Heintzmann, T. M. Jovin, C. Cremer, "Saturated patterned excitation microscopy-a concept for optical resolution improvement." J Opt Soc Am A Opt Image Sci Vis. 19:1599-609 (2002).

8. M. G. Gustafsson, "Nonlinear structured-illumination microscopy: Wide-field fluorescence imaging with theoretically unlimited resolution," Proc. Natl. Acad. Sci. USA 102, 13081-13086 (2005).

9. E. Betzig, G. H. Patterson, R. Sougrat et al., "Imaging intracellular fluorescent proteins at nanometer resolution," Science 313, 1642-1645 (2006).

10. S. T. Hess, T. P. Girirajan, M. D. Mason, "Ultrahigh resolution imaging by fluorescence photoactivation localization microscopy," Biophys. J. 91, 4258-4272 (2006).

11. M. J. Rust, M. Bates, X. Zhuang, "Sub-diffractionlimit imaging by stochastic optical reconstruction microscopy (STORM)." Nat. Methods. 3, 793-795 (2006).

12. D. Axelrod, "Total internal reflection fluorescence microscopy in cell biology," Traffic. 2, 764-774 (2001).

13. S. M. Simon, "Partial internal reflections on total internal reflection fluorescent microscopy," Trends. Cell. Biol. 19, 661-668 (2009).

14. D. Yarar, C. M. Waterman-Storer, S. L. Schmid, "A dynamic actin cytoskeleton functions at multiple stages of clathrin-mediated endocytosis," Mol. Biol. Cell. 16, 964-975 (2005).

15. J. Z. Rappoport, "Focusing on clathrin-mediated endocytosis," Biochem. J. 412, 415-423 (2008).

16. J. Z. Rappoport, S. M. Simon, "Endocytic trafficking of activated EGFR is AP-2 dependent and occurs through preformed clathrin spots," J. Cell. Sci. 122, 1301-1305 (2009).

17. T. Yuan, L. Liu, Y. Zhang et al., "Diacylglycerol guides the hopping of clathrin-coated pits along microtubules for exo-endocytosis coupling," Dev Cell. 35, 120-130 (2015).

18. J. Boulanger, C. Gueudry, D. Munch et al., "Fast high-resolution 3D total internal reflection fluorescence microscopy by incidence angle scanning and azimuthal averaging," Proc. Natl. Acad. Sci. USA 111, 17164-171649 (2014).

19. D. Li, K. Herault, K. Zylbersztejn et al., "Astrocyte VAMP3 vesicles undergo Ca2+-independent cycling and modulate glutamate transporter trafficking," J. Physiol. 593, 2807-2832 (2015).

20. S. Saffarian, T. Kirchhausen, "Differential evanescence nanometry: Live-cell fluorescence measurements with 10-nm axial resolution on the plasma membrane," Biophys. J. 94, 2333-2342 (2008).

21. W. T. Pitkeathly, N. S. Poulter, E. Claridge et al., "Auto-align - multi-modality fluorescence microscopy image co-registration," Traffic. 13, 204-217 (2012).
22. T. A. Klar, S. W. Hell, "Subdiffraction resolution in far-field fluorescence microscopy," Opt. Lett. 24, 954-956 (1999).

23. T. Grotjohann, I. Testa, M. Leutenegger et al., "Diffraction-unlimited all-optical imaging and writing with a photochromic GFP," Nature 478, 204208 (2011).

24. R. J. Marsh, S. Culley, A. J. Bain, "Low power super resolution fluorescence microscopy by lifetime modification and image reconstruction," Opt. Express. 22, 12327-12338 (2014).

25. C. F. Kuang, S. Li, W. Liu et al., "Breaking the diffraction barrier using fluorescence emission difference microscopy." Sci. Rep. 3, (2013).

26. W. Yu, Z. Ji, D. Dong, X. Yang, Y. Xiao, Q. Gong, P. Xi, K. Shi, "Super-resolution deep imaging with hollow Bessel beam STED microscopy," Laser Photon. Rev. 10, 147-152 (2016).

27. X. Yang, H. Xie, E. Alonas et al., "Mirror-enhanced super-resolution microscopy," Light Sci Appl. 5, (2016).

28. K. I. Willig, R. R. Kellner, R. Medda et al., "Nanoscale resolution in GFP-based microscopy," Nat. Methods. 3, 721-723 (2006).

29. M. Ishigaki, M. Iketani, M. Sugaya et al., "STED superresolution imaging of mitochondria labeled with TMRM in living cells," Mitochondrion 28, 79-87 (2016).

30. D. C. Jans, C. A. Wurm, D. Riedel et al., "STED super-resolution microscopy reveals an array of MINOS clusters along human mitochondria," Proc. Natl. Acad. Sci. USA 110, 8936-8941 (2013).

31. E. D'Este, D. Kamin, C. Velte et al., "Subcortical cytoskeleton periodicity throughout the nervous system," Sci. Rep. 6, 22741 (2016).

32. E. D'Este, D. Kamin, F. Gottfert et al., "STED nanoscopy reveals the ubiquity of subcortical cytoskeleton periodicity in living neurons," Cell Rep. 10, 1246-1251 (2015).

33. H. Nishimune, Y. Badawi, S. Mori et al., "Dual-color STED microscopy reveals a sandwich structure of Bassoon and Piccolo in active zones of adult and aged mice," Sci. Rep. 6, 27935 (2016).

34. F. Bottanelli, E. B. Kromann, E. S. Allgeyer et al., "Two-colour live-cell nanoscale imaging of intracellular targets." Nat. Commun. 7, 10778 (2016).

35. L. Meyer, D. Wildanger, R. Medda et al., "Dualcolor STED microscopy at $30-\mathrm{nm}$ focal-plane resolution," Small. 4, 1095-1100 (2008).

36. S. C. Sidenstein, E. D'Este, M. J. Bohm et al., "Multicolour Multilevel STED nanoscopy of Actin/ Spectrin Organization at Synapses," Sci Rep. 6, 26725 (2016).

37. J. Buckers, D. Wildanger, G. Vicidomini et al., "Simultaneous multi-lifetime multi-color STED imaging for colocalization analyses," Opt. Express. 19, 3130-3143 (2011). 
38. D. Wildanger, R. Medda, L. Kastrup et al., "A compact STED microscope providing 3D nanoscale resolution," J. Microsc. 236, 35-43 (2009).

39. Y. Wu, X. Wu, R. Lu et al., "Resonant scanning with large field of view reduces photobleaching and enhances fluorescence yield in STED microscopy," Sci. Rep. 5, 14766 (2015).

40. D. Dan, M. Lei, B. Yao et al., "DMD-based LEDillumination super-resolution and optical sectioning microscopy," Sci. Rep. 3, 1116 (2013).

41. E. H. Rego, L. Shao, J. J. Macklin et al., "Nonlinear structured-illumination microscopy with a photoswitchable protein reveals cellular structures at 50nm resolution," Proc. Natl. Acad. Sci. USA 109, E135-E143 (2012).

42. D. Li, L. Shao, B. C. Chen et al., "Advanced Imaging. Extended-resolution structured illumination imaging of endocytic and cytoskeletal dynamics," Science 349, aab3500 (2015).

43. H. Gong, D. Xu, J. Yuan et al., "High-throughput dual-colour precision imaging for brain-wide connectome with cytoarchitectonic landmarks at the cellular level," Nat. Commun. 7, 12142 (2016).

44. C. Li, H. Yan, L. X. Zhao et al., "A trident dithienylethene-perylenemonoimide dyad with super fluorescence switching speed and ratio," Nat. Commun. 5, 5709 (2014).

45. D. Pan, Z. Hu, F. Qiu et al., "A general strategy for developing cell-permeable photo-modulatable organic fluorescent probes for live-cell super-resolution imaging," Nat. Commun. 5, 5573 (2014).

46. B. Huang, W. Wang, M. Bates et al., "Three-dimensional super-resolution imaging by stochastic optical reconstruction microscopy," Science $\mathbf{3 1 9}$, 810-813 (2008).

47. A. N. Boettiger, B. Bintu, J. R. Moffitt et al., "Super-resolution imaging reveals distinct chromatin folding for different epigenetic states," Nature 529, 418-422 (2016).

48. M. Lakadamyali, "Super-resolution microscopy: Going live and going fast," Chemphyschem 15, 630636 (2014).

49. Z. Liu, D. Xing, Q. P. Su et al., "Super-resolution imaging and tracking of protein-protein interactions in sub-diffraction cellular space," Nat. Commun. 5, 4443 (2014).

50. M. Bates, S. A. Jones, X. Zhuang, "Preparation of photoswitchable labeled antibodies for STORM imaging," Cold Spring Harb Protoc. 2013, 540-541 (2013).

51. M. Bates, S. A. Jones, X. Zhuang, "Transfection of genetically encoded photoswitchable probes for STORM imaging," Cold Spring Harb Protoc. 2013, 537-539 (2013).
52. L. Zhu, W. Zhang, D. Elnatan et al., "Faster STORM using compressed sensing," Nat. Methods. 9, 721-723 (2012).

53. F. Mark, W. Barry, A. P. Michael, Neuroscience: Exploring the Brain, Nishimura Co. Ltd., Japan, pp. 144-152 (2007).

54. K. I. Willig, S. O. Rizzoli, V. Westphal et al., "STED microscopy reveals that synaptotagmin remains clustered after synaptic vesicle exocytosis," Nature 440, 935-939 (2006).

55. J. J. Sieber, K. I. Willig, C. Kutzner et al., "Anatomy and dynamics of a supramolecular membrane protein cluster," Science 317, 1072-1076 (2007).

56. V. Westphal, S. O. Rizzoli, M. A. Lauterbach et al., "Video-rate far-field optical nanoscopy dissects synaptic vesicle movement," Science 320, 246-249 (2008).

57. S. Nofal, U. Becherer, D. Hof et al., "Primed vesicles can be distinguished from docked vesicles by analyzing their mobility," J. Neurosci. 27, 1386-1395 (2007).

58. Y. Gu, R. L. Huganir, "Identification of the SNARE complex mediating the exocytosis of NMDA receptors," Proc. Natl. Acad. Sci. USA 113, 1228012285 (2016).

59. U. V. Nagerl, K. I. Willig, B. Hein et al., "Live-cell imaging of dendritic spines by STED microscopy," Proc. Natl. Acad. Sci. USA 105, 18982-18987 (2008).

60. M. Schouten, G. M. De Luca, D. K. Alatriste Gonzalez et al., "Imaging dendritic spines of rat primary hippocampal neurons using structured illumination microscopy," J. Vis. Exp. (2014).

61. G. Zhong, J. He, R. Zhou et al., "Developmental mechanism of the periodic membrane skeleton in axons," Elife. 3, (2014).

62. K. Xu, G. Zhong, X. Zhuang, "Actin, spectrin, and associated proteins form a periodic cytoskeletal structure in axons," Science 339, 452-456 (2013).

63. K. Zhanghao, L. Chen, X. Yang et al., "Super-resolution dipole orientation mapping via polarization demodulation," Nature 5, e16166 (2016).

64. K. Wang, D. E. Milkie, A. Saxena et al., "Rapid adaptive optical recovery of optimal resolution over large volumes," Nat. Methods. 11, 625-628 (2014).

65. K. Si, R. Fiolka, M. Cui, "Fluorescence imaging beyond the ballistic regime by ultrasound pulse guided digital phase conjugation," Nat. Photonics. 6, 657-661 (2012).

66. N. Ji, D. E. Milkie, E. Betzig, "Adaptive optics via pupil segmentation for high-resolution imaging in biological tissues," Nat. Methods. 7, 141-147 (2010).

67. K. Xu, H. P. Babcock, X. Zhuang, "Dual-objective STORM reveals three-dimensional filament organization in the actin cytoskeleton," Nat. Methods. 9, 185-188 (2012). 
68. J. H. Resau, Handbook of Biological Confocal Microscopy, Springer, US (2006).

69. P. Kner, B. B. Chhun, E. R. Griffis et al., "Superresolution video microscopy of live cells by structured illumination," Nat. Methods. 6, 339-342 (2009).
70. Y. Fu, P. W. Winter, R. Rojas et al., "Axial superresolution via multiangle TIRF microscopy with sequential imaging and photobleaching," Proc. Natl. Acad. Sci. USA 113, 4368-4373 (2016). 